\title{
ALGEBRAIC STEIN VARIETIES
}

\author{
JING ZHANG
}

\begin{abstract}
It is well-known that the associated analytic space of an affine variety defined over $\mathbb{C}$ is Stein but the converse is not true, that is, an algebraic Stein variety is not necessarily affine. In this paper, we give sufficient and necessary conditions for an algebraic Stein variety to be affine. One of our results is that an irreducible quasiprojective variety $Y$ defined over $\mathbb{C}$ with dimension $d(d \geq 1)$ is affine if and only if $Y$ is Stein, $H^{i}\left(Y, \mathcal{O}_{Y}\right)=0$ for all $i>0$ and $\kappa(D, X)=d$ (i.e., $D$ is a big divisor), where $X$ is a projective variety containing $Y$ and $D$ is an effective divisor with support $X-Y$. If $Y$ is algebraic Stein but not affine, we also discuss the possible transcendental degree of the nonconstant regular functions on $Y$. We prove that $Y$ cannot have $d-1$ algebraically independent nonconstant regular functions. The interesting phenomenon is that the transcendental degree can be even if the dimension of $Y$ is even and the degree can be odd if the dimension of $Y$ is odd.
\end{abstract}

\section{Introduction}

We work over complex number field $\mathbb{C}$ and use the terminology in Hartshorne's book [H1].

Affine varieties (i.e., irreducible closed subsets of $\mathbb{C}^{n}$ in Zariski topology) are important in algebraic geometry. Since J.-P. Serre discovered his well-known cohomology criterion ([H2], Chapter II, Theorem 1.1), the criteria for affineness have been investigated by many algebraic geometers (Goodman and Harshorne [GH]; Hartshorne [H2], Chapter II; Kleiman [Kl]; Neeman [N]; Zhang[Zh3]). Corresponding to affine varieties in algebraic geometry, in complex geometry, Stein varieties hold similar important position. A complex space $Y$ is Stein if and only if $H^{i}(Y, G)=0$ for every analytic coherent sheaf $G$ on $Y$ and all positive integers $i$. Or equivalently, if $Y$ is a complex analytic variety, then $Y$ is Stein if and only if it is both holomorphically convex and holomorphically separable ([Gu], Page 143). We say that $Y$ is holomorphically convex if for any discrete sequence $\left\{y_{n}\right\} \subset Y$, there is a holomorphic function $f$ on $Y$ such that the supremum of the set $\left\{\left|f\left(y_{n}\right)\right|\right\}$ is $\infty$. $Y$ is holomorphically separable if for every pair $x, y \in Y, x \neq y$, there is a holomorphic function $f$ on $Y$ such that $f(x) \neq f(y)$.

It is well-known that the associated analytic space of an affine variety defined over $\mathbb{C}$ is Stein ([H2], Chapter VI, Proposition 3.1), but the converse is not true, that is, an algebraic Stein variety is not necessarily affine. (An algebraic variety is a quasi-projective variety, that is, a Zariski open subset of a projective variety.) The reason is that there are algebraic varieties which have plenty of global holomorphic functions but do not have any nonconstant regular functions. J.-P. Serre constructed the first example, an algebraic surface which is Stein but not affine ([H2], Chapter

Received by the editors April 10, 2007.

2000 Mathematics Subject Classification. 14J10, 14J40, 32E10, 32Q28. 
VI, Example 3.2). Based on Serre's construction and the Künneth formula [SaW], it is easy to construct higher dimensional algebraic Stein varieties which are not affine.

Then naturally we would ask: What is the necessary and sufficient condition for an algebraic Stein variety to be affine? We will answer this question by showing the following Theorem 1.1 and Theorem 1.2. In 1988, Neeman proved that a quasi-affine normal variety $U$ of finite type over $\mathbb{C}$ is affine if $U$ is Stein and the $\operatorname{ring} \Gamma\left(U, \mathcal{O}_{U}\right)$ of regular functions on $U$ is a finitely generated $\mathbb{C}$-algebra $([\mathrm{N}]$, Proposition 5.5). A quasi-affine variety is a Zariski open subset of an affine variety contained in $\mathbb{C}^{n}$ for some positive integer $n$. So his theorem is a nice local result. Our contribution is to give global criteria which work for any algebraic Stein variety $Y$. We do not assume that $Y$ is contained in $\mathbb{C}^{n}$.

Let $Y$ be an irreducible quasi-projective variety and $X$ be an irreducible projective variety containing $Y$. By further blowing up the closed subset of the boundary $X-Y$, we may assume that $X-Y$ is of pure codimension 1 and is support of an effective divisor $D$ with simple normal crossings. Let $\kappa(D, X)$ be the $D$-dimension (or Iitaka dimension) ([I2], Lecture 3$)$ of $X$, then the Kodaira dimension $\kappa(X)$ is $\kappa\left(K_{X}, X\right)$, where $K_{X}$ is the canonical divisor of $X$. We will use these notations throughout this paper. In particular, all sheaves are algebraic and coherent.

By definition, $\kappa(D, X)$ measures the number of algebraically independent nonconstant regular functions on $Y$. Since $D$ is effective, the dimension of linear space $H^{0}\left(X, \mathcal{O}_{X}(m D)\right)$, denoted by $h^{0}\left(X, \mathcal{O}_{X}(m D)\right)$, is not zero. Therefore we always have $\kappa(D, X) \geq 0$.

Theorem 1.1. An irreducible algebraic Stein surface $Y$ is affine if and only if $\kappa(D, X)=2$.

Thanks for the referee's comment about Theorem 1.1. The referee pointed out that Theorem 1.1 may not be new and the proof is standard. We wrote it separately because of the following reasons. First, we cannot find any reference and in surface case, both the condition and proof are different from higher dimensional case. In Theorem 1.1, we do not need the condition $H^{i}\left(Y, \mathcal{O}_{Y}\right)=0$ for all $i>0$ as in Theorem 1.2 for higher dimensional varieties, where $\mathcal{O}_{Y}$ is the sheaf of regular functions on $Y$. Finally, the surface case is an initial step therefore is also important because our proof for higher dimensional varieties is based on mathematical induction.

Theorem 1.2. An irreducible algebraic Stein variety $Y$ of dimension $d \geq 1$ is affine if and only if $\kappa(D, X)=d$ and $H^{i}\left(Y, \mathcal{O}_{Y}\right)=0$ for all $i>0$.

Theorem 1.3. If $Y$ is an irreducible algebraic Stein variety with dimension $d \geq 1$, then

(1) $\kappa(D, X) \neq d-1$;

(2) If $d=2 k$, then $\kappa(D, X)$ can be any even number $i, i=0,2,4, \ldots, 2 k$;

(3) If $d=2 k+1$, then $\kappa(D, X)$ can be any odd number $j, j=1,3,5, \ldots, 2 k-1,2 k+1$.

Definition 1.4. An algebraic variety $Y$ is regularly separable if for any two distinct points $y_{1}$ and $y_{2}$ on $Y$, there is a regular function $f$ on $Y$ such that $f\left(y_{1}\right) \neq f\left(y_{2}\right)$.

Corollary 1.5. If $Y$ is an irreducible algebraic Stein variety with dimension $d \geq 1$, then the following conditions are equivalent

(1) $Y$ is affine; 
(2) $Y$ is regularly separable and $H^{i}\left(Y, \mathcal{O}_{Y}\right)=0$ for all $i>0$;

(3) $Y$ is regularly separable and $\Gamma\left(Y, \mathcal{O}_{Y}\right)$ (the ring of regular functions on $Y$ ) is a finitely generated $\mathbb{C}$-algebra;

(4) $\kappa(D, X)>\max (1, d-2)$ and $H^{i}\left(Y, \mathcal{O}_{Y}\right)=0$ for all $i>0$;

(5) $\kappa(D, X)=d$, and $H^{i}\left(Y, \mathcal{O}_{Y}\right)=0$ for all $i>0$.

This paper is organized as follows. In Section 2, we will prove Theorem 1.1 and some results for surfaces. In Section 3, we will prove Theorem 1.2, Theorem 1.3 and Corollary 1.5.

\section{Surfaces}

A fiber space is a proper surjective morphism $f: V \rightarrow W$ between two varieties $V$ and $W$ such that the general fiber is connected.

$D$ is a big divisor if $\kappa(D, X)=d$, where $d$ is the dimension of $X$. If $L$ is a line bundle on a projective manifold $M$, it determines a Cartier divisor $D$. We define $\kappa(L, M)=\kappa(D, M)$.

Theorem 2.1 (Fujita). Let $M$ and $S$ be two projective manifolds. Let $\pi: M \rightarrow S$ be a fiber space and let $L$ and $H$ be line bundles on $M$ and $S$ respectively. Suppose that $\kappa(H, S)=\operatorname{dim} S$ and that $\kappa\left(a L-b \pi^{*}(H)\right) \geq 0$ for certain positive integers $a, b$. Then $\kappa(L, M)=\kappa\left(\left.L\right|_{F}, F\right)+\kappa(H, S)$ for a general fiber $F$ of $\pi$.

We can freely adjust the coefficients of the effective divisor $D$ or blow up a closed subvariety in $D$ (and still denote the divisor as $D$ ) without changing the $D$-dimension because of the following two properties ([I1]; [Uen], Chapter II, Section 5).

Let $f: X^{\prime} \rightarrow X$ be a surjective morphism between two complete varieties $X^{\prime}$ and $X$. Let $D$ be a divisor on $X$ and $E$ an effective divisor on $X^{\prime}$ such that $\operatorname{codim} f(E) \geq 2$, then

$$
\kappa\left(f^{-1}(D)+E, X^{\prime}\right)=\kappa(D, X),
$$

where $f^{-1}(D)$ is the reduced transform of $D$, defined to be $f^{-1}(D)=\sum D_{i}, D_{i}$ 's are the irreducible components of $D$. The $D$-dimension also does not depend on the coefficients of $D$ if $D \geq 0$. Precisely, let $D_{1}, D_{2}, \cdots, D_{n}$ be any divisor on $X$ such that for every $i, 1 \leq i \leq n, \kappa\left(D_{i}, X\right) \geq 0$, then for integers $p_{1}>0, \cdots, p_{n}>0$, we have [I3],

$$
\kappa\left(D_{1}+\cdots+D_{n}, X\right)=\kappa\left(p_{1} D_{1}+\cdots+p_{n} D_{n}, X\right) .
$$

In particular, if $D_{i}$ 's are irreducible components of $D$ and $D$ is effective, then we can change its coefficients to different nonzero positive integers but do not change the $D$-dimension.

The following Lemma 2.2 is a modification of Goodman's theorem ([H2], Chapter 2, Theorem 4.2). The advantage of our $D$-dimension version is that we can generalized it to higher dimensional case [Zh3], whereas Goodman's surface theorem ([H2], Theorem 4.2 ) is not true for higher dimensional varieties: If $Y$ is a higher dimensional affine variety contained in a projective variety $X$, then the boundary $X-Y$ may not be the support of any ample divisor on $X$. Goodman proved that only after further blowing up the closed subset on the boundary, the new boundary may be the support of an ample divisor. The partial reason is that the intersection theory for higher dimensional varieties is much more complicated and generally an effective big divisor has no Zariski decomposition if the dimension is higher than 2. 
A divisor $D$ of $X$ is nef if for every irreducible curve $C$ on $X$, we have $D \cdot C \geq 0$.

Lemma 2.2. Let $Y$ be an irreducible open smooth algebraic surface. Let $X$ be a smooth projective surface containing $Y$. Then $Y$ is affine if and only if the following three conditions hold

(1) $Y$ contains no complete curves;

(2) The boundary $X-Y$ is connected;

(3) $\kappa(D, X)=2$, where $D$ is an effective divisor with support $X-Y$.

Proof. We will prove that the boundary $X-Y$ is the support of an ample divisor.

Since $Y$ contains no complete curves, the boundary $X-Y$ cannot be blown down to a point. And $X-Y$ is of pure codimension 1 since it is connected.

Write the Zariski decomposition $D=P+N$, where $N$ is effective and negative definite, $P$ is effective and nef and any prime component of $N$ does not intersect $P$ [Za]. We may assume that both $P$ and $N$ are integral by multiplying a positive integer to the equation since both $P$ and $N$ are $\mathbb{Q}$ divisors $(D$ is a Weil divisor but $P$ and $N$ may have rational coefficients [Za]). Let $\operatorname{Supp} D=\left\{D_{1}, D_{2}, \cdots, D_{n}\right\}=X-Y$. Since $\kappa(D, X)=2, P^{2}>0$ ([Sa1]; [Ba], Corollary 14.18). First we claim that $\operatorname{Supp} P=\operatorname{Supp} D=X-Y$. If $\operatorname{Supp} P \neq X-Y$, then there is a prime component, say $D_{1}$, in $X-Y$ such that $P \cdot D_{1}>0$ and $D_{1}$ is not a component of $P$ since $X-Y$ is connected. Let

$$
Q=m P+D_{1},
$$

where $m$ is a big positive integer. Then $Q$ is an effective divisor and $\operatorname{Supp} Q=$ $\operatorname{Supp} P \cup D_{1}$. Since $P^{2}>0$, we may choose $m$ such that

$$
Q^{2}=m^{2} P^{2}+2 m P \cdot D_{1}+D_{1}^{2}>0 .
$$

For every prime component $E$ in $P$, since $P$ is nef and $D_{1}$ is not contained in $\operatorname{Supp} P$, for sufficiently large $m$, we have

$$
Q \cdot E=m P \cdot E+D_{1} \cdot E \geq 0, \quad D_{1} \cdot Q=m D_{1} \cdot P+D_{1}^{2}>0 .
$$

Since $Y$ contains no complete curves, any irreducible complete curve outside $X-Y$ intersects $X-Y$. Thus we get a new effective divisor $Q$ such that $Q$ is nef and $Q^{2}>0$. We may replace $P$ by $Q$ and still call it $P$. By finitely many such replacements, we can find an effective nef divisor $P$ such that $P^{2}>0$ and $\operatorname{Supp} P=\operatorname{Supp} D=X-Y$.

We claim that the boundary $X-Y$ is the support of an ample divisor. In fact, the following three conditions imply the ampleness:

(1) $X-Y$ is connected;

(2) $Y$ contains no complete curves;

(3) There is an effective nef divisor $P$ with $\operatorname{supp} P=X-Y$ and $P^{2}>0$.

There is a proof for the above claim in [H2], page 69-71. It is also not hard to prove it by computing intersection numbers (we may change the coefficients if necessary).

Q.E.D.

Notice that the above lemma holds for complete normal surfaces. For a complete normal surface $X$, the intersection theory is due to Mumford [Mu2]. Let $\operatorname{Div}(X)$ be the group of Weil divisors of $X$. Let $\operatorname{Div}(X, \mathbb{Q})=\operatorname{Div}(X) \otimes \mathbb{Q}$ be the group of $\mathbb{Q}$-divisors. The intersection pairing

$$
\operatorname{Div}(X, \mathbb{Q}) \times \operatorname{Div}(X, \mathbb{Q}) \rightarrow \mathbb{Q}
$$


is defined in the following way. Let $\pi: X^{\prime} \rightarrow X$ be a resolution and let $A=\cup E_{i}$ denote the exceptional set of $\pi$. For a $\mathbb{Q}$-divisor $D$ on $X$ we define the inverse image $\pi^{*} D$ as

$$
\pi^{*} D=\bar{D}+\sum a_{i} E_{i}
$$

where $\bar{D}$ is the strict transform of $D$ by $\pi$ and the rational numbers $a_{i}$ are uniquely determined by the equations $\bar{D} \cdot E_{j}+\sum a_{i} E_{i} \cdot E_{j}=0$ for all $j$. For two divisors $D$ and $D^{\prime}$ on $X$, their intersection number is defined to be

$$
D \cdot D^{\prime}=\pi^{*} D \cdot \pi^{*} D^{\prime} \text {. }
$$

Lemma 2.3. [Fujita] Let $D$ be an effective $\mathbb{Q}$-divisor on a normal projective surface $X$. Then there exists a unique decomposition

$$
D=P+N
$$

satisfying the following conditions:

(1) $N$ is an effective $\mathbb{Q}$-divisor and either $N=0$ or the intersection matrix of the irreducible components of $N$ is negative definite;

(2) P is a nef $\mathbb{Q}$-divisor and the intersection of $P$ with each irreducible component of $N$ is zero.

Lemma 2.4. Let $Y$ be an irreducible algebraic surface. Let $X$ be a projective surface containing $Y$. Then $Y$ is affine if and only if the following three conditions hold

(1) $Y$ contains no complete curves;

(2) The boundary $X-Y$ is connected;

(3) $\kappa(D, X)=2$, where $D$ is an effective divisor with support $X-Y$.

Proof. If we have a surjective and finite morphism from a variety $Y^{\prime}$ to $Y$, then $Y$ is affine if and only if $Y^{\prime}$ is affine by Chevalley's theorem ([H2], Chapter 2, Corollary 1.5). Thus $Y$ is affine if and only if its normalization is affine. So we may assume that both $Y$ and $X$ are normal by taking their normalization. On a normal projective surface, the intersection theory and Zariski decomposition remain true by Lemma 2.3 [Mu2, Sa2]. Therefore Lemma 2.2 holds for normal projective surfaces. In fact, write the Zariski decomposition $D=P+N$ by Lemma 2.3, then $P^{2}>0$ ([Ba], Corollary 14.18, Page 222). By the same argument as in the proof of Lemma 2.2, we can find a new effective nef divisor, still denoted by $P$, such that $\operatorname{supp} P=X-Y$. By changing the coefficients of $P$, we can find an ample divisor supported in $X-Y$.

Q.E.D.

Remark 2.5. Lemma 2.4 does not hold for threefolds. If $Y$ is a smooth algebraic threefold without complete curves and the boundary $X-Y$ is connected for a smooth completion $X$ of $Y$, then we cannot claim that $Y$ is affine by the following two conditions:

(1) The boundary $X-Y$ is of pure codimension 1;

(2) $\kappa(D, X)=3$.

The reason is that in surface case, the two conditions, i.e., $Y$ contains no complete curves and $X-Y$ is connected, imply that for any smooth completion $Z$ (may be different from $X$ ) of $Y$, the boundary $Z-Y$ is again of pure codimension 1 . This is of course not true in higher dimension. For instance, remove a hyperplane section 
$H$ and a line $L$ from $\mathbb{P}^{3}$, where $L$ is not contained in $H$. Let $Y=\mathbb{P}^{3}-H-L$. Then $Y$ contains no complete curves. Let $f: X \rightarrow \mathbb{P}^{3}$ be the blowing up of $\mathbb{P}^{3}$ along $L$. Then $X$ is a smooth projective threefold and $Y$ is an open subset of $X$. Let $D=f^{-1}(H)+E$, where $E$ is the exceptional divisor. Then by the properties of $D$-dimension, $\kappa(D, X)=\kappa\left(H, \mathbb{P}^{3}\right)=3$. But $Y$ is not affine since the boundary $\mathbb{P}^{3}-Y$ is not of pure codimension 1 ([H2], Chapter 2, Proposition 3.1).

Corollary 2.6. Suppose that we have a surjective morphism from an irreducible smooth algebraic surface $Y$ to a smooth affine curve $C$. Let $X$ be a smooth projective surface containing $Y$. If $Y$ contains no complete curves and the boundary $X-Y$ is connected, then $Y$ is affine.

Proof. Let $f: Y \rightarrow C$ be the given morphism. Then $f$ gives a rational map from $X$ to $\bar{C}$, where $\bar{C}$ is the smooth completion of $C$. Resolve the indeterminacy of $f$ on the boundary $X-Y$. We may replace $X$ by its suitable blowing up and assume that $f: X \rightarrow \bar{C}$ is a surjective and proper morphism. Notice that this procedure does not change $Y$. $Y$ is still an open subset of $X$. By Stein factorization, we may assume that every fiber is connected and general fiber is smooth. Pick a point $t_{1} \in \bar{C}-C$, then

$$
h^{1}\left(\bar{C}, \mathcal{O}_{\bar{C}}\left(n t_{1}\right)\right)=0
$$

since $n t_{1}$ is ample for large $n$ ([H1], Chapter IV, Corollary 3.3). By the Riemann-Roch formula,

$$
h^{0}\left(\bar{C}, \mathcal{O}_{\bar{C}}\left(n t_{1}\right)\right)=1+n-g(\bar{C}) .
$$

So $\kappa\left(t_{1}, \bar{C}\right)=1$ ([Uen], Chapter II, Section 5 ). For a general point $t \in C$, we may assume that the fiber $f^{-1}(t)$ determined by $t$ is smooth ([Uen], Corollary 1.8). By Riemann-Roch formula, there is a positive integer $m$, such that $h^{0}\left(\bar{C}, \mathcal{O}_{\bar{C}}\left(m t_{1}-t\right)\right)>$ 1. Let $s$ be a nonconstant section of $H^{0}\left(\bar{C}, \mathcal{O}_{\bar{C}}\left(m t_{1}-t\right)\right)$, then

$$
\operatorname{div} s+m t_{1}-t \geq 0 \text {. }
$$

Pull it back to $X$, we have

$$
f^{*}\left(\operatorname{div} s+m t_{1}-t\right)=\operatorname{div} f^{*}(s)+m f^{*}\left(t_{1}\right)-f^{*}(t) \geq 0 .
$$

Let $D_{1}=f^{*}\left(t_{1}\right)$ and $F=f^{*}(t)$, then $f^{*}(s)$ is a nonconstant section of $H^{0}\left(X, \mathcal{O}\left(m D_{1}-F\right)\right)$. So

$$
h^{0}\left(X, \mathcal{O}\left(m D_{1}-F\right)\right)>1
$$

Choose an effective divisor $D$ with support $X-Y$ such that $D_{1} \leq D$, then we have

$$
h^{0}(X, \mathcal{O}(m D-F)) \geq h^{0}\left(X, \mathcal{O}\left(m D_{1}-F\right)\right)>1 .
$$

Since $\left.F\right|_{Y}$ is a smooth affine curve ([H2], Chapter 2, Proposition 4.1), and $F$ intersects $D$ on the boundary $X-Y,\left.D\right|_{F}$ is an effective divisor on $F$. So

$$
h^{0}\left(F, \mathcal{O}_{F}\left(\left.m D\right|_{F}\right)\right) \geq m+1-g(F) .
$$

Therefore $\kappa\left(\left.D\right|_{F}, F\right)=1$ ([Uen], Chapter II, Section 5). By Theorem 2.1,

$$
\kappa(D, X)=\kappa(m D, X)=\kappa\left(\left.m D\right|_{F}, F\right)+\kappa(t, \bar{C})=2
$$

By Lemma 2.2, $Y$ is affine.

Q.E.D.

It is easy to see that we can drop the smooth condition in Corollary 2.6. 
Remark 2.7. Corollary 2.6 does not hold for threefolds. We have the following counterexample.

Let $C_{t}$ be a smooth projective elliptic curve defined by $y^{2}=x(x-1)(x-t)$, $t \neq 0,1$. Let $Z$ be the elliptic surface defined by the same equation. We have surjective morphism from $Z$ to $C=\mathbb{C}-\{0,1\}$ such that for every $t \in C$, the fiber $f^{-1}(t)=C_{t}$. In [Zh2], we proved the following two claims.

1. There is a rank 2 vector bundle $E$ on $Z$ such that when restricted to $C_{t}$, $\left.E\right|_{C_{t}}=E_{t}$ is the unique nonsplit extension of $\mathcal{O}_{C_{t}}$ by $\mathcal{O}_{C_{t}}$, where $f$ is the morphism from $Z$ to $C$.

2. There is a divisor $D$ on $X=\mathbb{P}_{Z}(E)$ such that when restricted to the ruled surface $X_{t}=\mathbb{P}_{C_{t}}\left(E_{t}\right),\left.D\right|_{X_{t}}=D_{t}$ is the canonical section of $X_{t}$.

Let $Y=X-D$, then $Y$ contains no complete curves since we have $H^{i}\left(Y, \Omega_{Y}^{j}\right)=0$ for all $i>0$ and $j \geq 0$ [Zh2]. And we have the morphism from $Y$ to $C$. But $Y$ is not affine since $Y$ has only one algebraically independent nonconstant regular function [Zh2]. I do not know whether $Y$ is Stein or not.

Theorem 2.8. Let $Y$ be a Stein algebraic surface contained in a projective surface $X$. Then $Y$ is affine if and only if $\kappa(D, X)=2$, where $D$ is an effective divisor with support $X-Y$. Moreover, if $Y$ is not affine, then $\kappa(D, X)=0$.

Proof. We may assume that $Y$ is normal as in the proof of Lemma 2.4. Since $Y$ is Stein, it contains no complete curves and the boundary is connected and of pure codimension 1 ([N], Proposition 3.4). Now the first claim is obvious by Lemma 2.4.

For the second claim, notice that if $\kappa(D, X)=1$, then we have surjective morphism from $Y$ to a smooth affine curve $C$. If $Y$ is smooth, by Corollary 2.6, $Y$ is affine which contradicts the fact $\kappa(D, X)=1$. If $Y$ is not smooth, let $\pi: X^{\prime} \rightarrow X$ be a birational proper surjective morphism such that $X^{\prime}$ is smooth, then by Corollary 2.6, $\kappa\left(\pi^{*} D, X^{\prime}\right)=2$. Therefore

$$
\kappa(D, X)=\kappa\left(\pi^{*} D, X^{\prime}\right)=2 .
$$

The maximum $D$-dimension implies that $Y$ is affine by Lemma 2.4 .

Since $\kappa(D, X) \geq 0$ ( $D$ is effective), if $Y$ is not affine, we have $\kappa(D, X)=0$.

Q.E.D.

We have finished the proof of Theorem 1.1.

Remark 2.9. If $Y$ is a smooth algebraic Stein variety with dimension 3 , then $\kappa(D, X) \neq$ 2 (the following Theorem 3.3) but $\kappa(D, X)=1$ is possible [Zh1, Zh2]. The case $\kappa(D, X)=0$ is a mystery. I do not know the existence of such algebraic variety. In surface case, J.-P. Serre gave an example ([H2], Chapter VI, Example 3.2): The open surface $Y$ is Stein and $\kappa(D, X)=0([\mathrm{Ku}]$, Lemma 1.8).

\section{Higher Dimensional Varieties}

Theorem 3.1. If $Y$ is an irreducible algebraic variety of dimension d, then $Y$ is affine if and only if $Y$ is Stein, $\kappa(D, X)=d$ and $H^{i}\left(Y, \mathcal{O}_{Y}\right)=0$ for all $i>0$, where $X$ is a completion of $Y, D$ is the effective divisor with support $X-Y$ (the boundary), and $\mathcal{O}_{Y}$ is the sheaf of regular functions on $Y$. 
Proof. One direction is trivial. If $Y$ is affine, then it is Stein ([H2], Chapter VI, Proposition 3.1) and $H^{i}\left(Y, \mathcal{O}_{Y}\right)=0$ for all $i>0$ by Serre's affineness criterion. The affineness of $Y$ also implies that $D$ is big since after further blowing up the boundary $X-Y$, it is the support of an ample divisor ([H2], Chapter 2, Section 6, Theorem $6.1)$.

We will prove the converse: $Y$ is affine if $Y$ is Stein, $\kappa(D, X)=d$ and $H^{i}\left(Y, \mathcal{O}_{Y}\right)=0$ for all $i>0$.

We may assume that $Y$ is normal since $Y$ is affine if and only if its normalization is affine. We also may assume $d>2$ since the case $d=1$ is trivial and the surface case has been proved in Section 2. We will use induction on the dimension of $Y$. Assume that the theorem holds for all $(d-1)$-dimensional irreducible varieties. We need to prove that the theorem holds for $d$-dimensional irreducible variety $Y$. The idea of proof is: with the inductive assumption, for any irreducible curve on $Y$, we can find a regular function on $Y$ such that when restricted to this curve, the function is not a constant. Then we can apply Goodman and Hartshorne's quasi-affineness criterion $[\mathrm{GH}]$ then Neeman's affineness criterion ([N], Theorem 4.1).

Step 1. Any prime principle divisor $Z=\left\{y \in Y, f(y)=0, f \in H^{0}\left(Y, \mathcal{O}_{Y}\right)\right\}$ on $Y$ satisfies the three conditions in Theorem 3.1.

Proof. $Z$ is Stein since it is a closed codimension 1 subvariety of the Stein variety $Y$. We have $H^{i}\left(Z, \mathcal{O}_{Z}\right)=0$ for all $i>0$ since $H^{i}\left(Y, \mathcal{O}_{Y}\right)=0$ for all $i>0$ and there is a short exact sequence

$$
0 \longrightarrow \mathcal{O}_{Y} \longrightarrow \mathcal{O}_{Y} \longrightarrow \mathcal{O}_{Z} \longrightarrow 0,
$$

where the first map is defined by $f$.

Let $\bar{Z}$ be the irreducible closed subvariety of codimension 1 on $X$ such that $Z$ is an open subset of $\bar{Z}$. Let $D_{0}=\left.D\right|_{\bar{Z}}$ be the restriction divisor on $\bar{Z}$. The boundary $\bar{Z}-Z$ is of pure codimension 1 on $\bar{Z}$ since $Z$ is Stein. We need to prove $\kappa\left(D_{0}, \bar{Z}\right)=d-1$.

First suppose that $Y$ and $X$ are smooth. The defining regular function $f$ of $Z$ on $Y$ gives a morphism from $Y$ to $\mathbb{C}$. Let $A=f(Y)$, then $A$ is a smooth affine curve. So $f$ defines a rational map from $X$ to $\mathbb{P}^{1}$. By Hironaka's elimination of points of indeterminacy of a rational map, we have a proper surjective morphism $f^{\prime}$ from a smooth projective variety $X^{\prime}$ to $\mathbb{P}^{1}$ such that $\left.f^{\prime}\right|_{Y}=f$ and $Y$ is still an open subset of $X^{\prime}$ (i.e., both $X^{\prime}$ and $X$ are smooth projective varieties containing $Y$ and $X^{\prime}$ can be blown down to $X$ ). We still denote the morphism by $f$ and $X^{\prime}$ by $X$. So we have the following commutative diagram

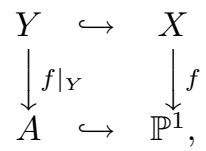

where $f$ is proper and surjective.

If the image of $D$ under $f$ is a set of finite points, then $Y$ contains complete varieties of codimension 1. This is not possible since $Y$ is Stein. Thus $f(D)=\mathbb{P}^{1}$. It is easy to see that $\bar{Z}$ is an irreducible component of the fiber $f^{-1}(0)$. By the property of $D$-dimension in Section 2, we may assume $\bar{Z}=f^{-1}(0)$. By Corollary 1.8, [Uen], a general fiber on $X$ is smooth.

Let $X \stackrel{h}{\longrightarrow} \bar{C} \stackrel{\alpha}{\longrightarrow} \mathbb{P}^{1}$ be the Stein factorization, then $\bar{C}$ is a smooth projective curve, where the first morphism $h$ from $X$ to $\bar{C}$ is proper and surjective and the second 
morphism $\alpha$ from $\bar{C}$ to $\mathbb{P}^{1}$ is a finite map such that every fiber of $h$ in $X$ is connected. Let $C=h(Y)$ be the image of $Y$ under the map $h$. Then $C$ is a smooth affine curve. The above commutative diagram becomes to

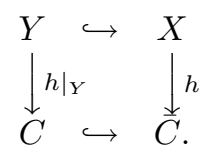

For any point $t \in C$, the corresponding open fiber $Y_{t}=h^{-1}(t) \cap Y$ is Stein since it is a closed subvariety of the Stein variety $Y$. Since $\bar{Z}=f^{-1}(0)$ is irreducible, $h^{-1}\left(\alpha^{-1}(0)\right)$ is also irreducible and

$$
h^{-1}\left(\alpha^{-1}(0)\right)=f^{-1}(0)=\bar{Z},
$$

where $\alpha^{-1}(0) \in C$.

Let $t$ be a general point of affine curve $C$. By Theorem 5.11, [Uen], let $X_{t}=h^{-1}(t)$, $D_{t}=\left.D\right|_{X_{t}}$, then we have

$$
d=\kappa(D, X) \leq \kappa\left(D_{t}, X_{t}\right)+1 \leq d .
$$

So $\kappa\left(D_{t}, X_{t}\right)=d-1$, for a general fiber $X_{t}$. By upper semi-continuity theorem,

$$
\kappa\left(D_{0}, \bar{Z}\right)=d-1 \text {. }
$$

If $Y$ is not smooth, we still have the commutative diagram

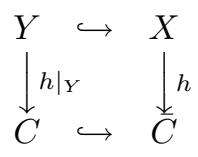

such that $h$ is proper, surjective and every fiber of $h$ in $X$ is connected. Let $\pi: X^{\prime} \rightarrow X$ be a proper surjective birational morphism such that $X^{\prime}$ is smooth, then we have a new commutative diagram

$$
\begin{array}{lll}
Y^{\prime} \hookrightarrow & X^{\prime} \\
\left.\downarrow g\right|_{Y} & & \mid g \\
C & \hookrightarrow & \stackrel{C}{C},
\end{array}
$$

where $g=h \circ \pi$ and $Y^{\prime}=\pi^{-1}(Y)$. Since $\pi$ is birational, the two function fields are isomorphic: $\mathbb{C}(X)=\mathbb{C}\left(X^{\prime}\right)$. Every fiber of $g$ is still connected since every fiber of $h$ is connected and $\mathbb{C}(X)=\mathbb{C}\left(X^{\prime}\right)$ ([Sh], Page 139, the function field $\mathbb{C}(\bar{C})$ is algebraically closed in $\left.\mathbb{C}\left(X^{\prime}\right)\right)$. So $\kappa\left(\pi^{*} D, X^{\prime}\right)=\kappa(D, X)=d$ and

$$
d=\kappa\left(\pi^{*} D, X^{\prime}\right) \leq \kappa\left(\left.\pi^{*} D\right|_{X_{a}^{\prime}}, X_{a}^{\prime}\right)+1 \leq d,
$$

where $X_{a}^{\prime}=g^{-1}(a)$ is the complete smooth fiber in $X^{\prime}$ ([Uen], Section 5) for a general point $a \in C$. Thus $\kappa\left(\left.\pi^{*} D\right|_{X_{a}^{\prime}}, X_{a}^{\prime}\right)=d-1$, which means that there are $d-1$ algebraically independent regular functions on $X_{a}^{\prime} \cap Y^{\prime}$. So $\pi_{*} X_{a}^{\prime} \cap Y=Y_{a}$ has $d-1$ algebraically independent regular functions, that is, $\kappa\left(D_{a}, X_{a}\right)=d-1$, where $X_{a}=h^{-1}(a)$ and $D_{a}=\left.D\right|_{X_{a}}$. In fact, we have

$$
\kappa\left(\left.D\right|_{X_{a}}, X_{a}\right)=\kappa\left(\pi^{*}\left(\left.D\right|_{X_{a}}\right), \pi^{-1}\left(X_{a}\right)\right)=\kappa\left(\left.\pi^{*} D\right|_{X_{a}^{\prime}}, X_{a}^{\prime}\right)=d-1 .
$$

By upper semi-continuity theorem, we are done. 
Step 2. If $H$ is a hyperplane such that $H$ intersects $X$ with an irreducible variety $\bar{Z}$, then the restriction of $\bar{Z}$ on $Y: Z=\left.\bar{Z}\right|_{Y}$ can be defined by a regular function $f$ on $Y$, that is, $Z=\left\{y \in Y, f(y)=0, f \in H^{0}\left(Y, \mathcal{O}_{Y}\right)\right\}$ is a prime principle divisor on $Y$.

Proof. Let $h$ be the homogeneous polynomial defining $H$. Let $H^{\prime}$ be a different hyperplane defined by a homogeneous polynomial $h^{\prime}$ such that $H^{\prime} \cap H$ is contained in the boundary $X-Y$. Then $h / h^{\prime}$ is a rational function on $X$, regular on $Y-H^{\prime}$ and defines $H \cap X=\bar{Z}$. Since $\kappa(D, X)=\operatorname{dim} X$, by the following lemma, any rational function on $X$ can be written as a quotient of two regular functions on $Y$. So $h / h^{\prime}=$ $f / g$, where both $f$ and $g$ are regular on $Y$. Therefore $Z=\{y \in Y, f(y)=0, f \in$ $\left.H^{0}\left(Y, \mathcal{O}_{Y}\right)\right\}$.

The following lemma is known and the proof can be found in [Mo].

Lemma 3.2. Let $X$ be normal proper over $\mathbb{C}$.

(1) If there is an $m_{0}>0$ such that for all $m>m_{0}, h^{0}\left(X, \mathcal{O}_{X}(m D)\right)>0$, then

$$
\mathbb{C}\left(\Phi_{|m D|}(X)\right)=Q((X, D)),
$$

where $\Phi_{|m D|}$ is the rational map from $X$ to a projective space defined by a basis of $H^{0}\left(X, \mathcal{O}_{X}(m D)\right)$.

(2) If $\kappa(D, X)=\operatorname{dim} X$, then $\Phi_{|m D|}$ is birational for all $m \gg 0$. In particular, $\mathbb{C}(X)=Q((X, D))$.

In the above lemma, $\mathbb{C}(X)$ is the function field of $X$. Let

$$
R(X, D)=\oplus_{\gamma=0}^{\infty} H^{0}\left(X, \mathcal{O}_{X}(\gamma D)\right)
$$

be the graded $\mathbb{C}$-domain and $R^{*} \subset R$ the multiplicative subset of all nonzero homogeneous elements. Then the quotient ring $R^{*-1} R$ is a graded $\mathbb{C}$-domain and its degree 0 part $\left(R^{*-1} R\right)_{0}$ is a field denoted by $Q((X, D))$, i.e.,

$$
Q((X, D))=\left(R^{*-1} R\right)_{0} .
$$

Step 3. For any irreducible curve $F$ on $Y$, there is a regular function on $Y$ such that the restriction of the function on $F$ is not a constant.

Proof. Let $\bar{F}$ be the irreducible complete curve on $X$ containing $F$ such that $\bar{F}-F$ is a set of finite points on the boundary $X-Y$.

By theorems of Seidenberg [Sei], away from the finite set $\bar{F}-F$, there is a hyperplane $H$ defined by an irreducible homogeneous polynomial $h$ such that $H \cap X$ is an irreducible and normal subvariety of codimension 1. Let $Z=H \cap Y$, then $Z$ is irreducible and normal. By Step 2, there is a regular function $f$ on $Y$ such that $Z$ is defined by $f$. We will prove that there is a regular function $r$ on $Y$ such that $\left.r\right|_{F}$ is not a constant.

Since $H$ is ample and $H$ does not contain any point of $\bar{F}-F$, the set $F \cap H=F \cap Z$ is not empty. If $F$ is not contained in $Z$, then $\left.f\right|_{F}$ is not a constant. Suppose that $F$ is a curve on $Z$.

By Step 1 and inductive assumption, $Z$ is affine. From the exact sequence

$$
0 \longrightarrow \mathcal{O}_{Y} \stackrel{f}{\longrightarrow} \mathcal{O}_{Y} \longrightarrow \mathcal{O}_{Z} \longrightarrow 0
$$

we have a surjective map from $H^{0}\left(Y, \mathcal{O}_{Y}\right)$ to $H^{0}\left(Z, \mathcal{O}_{Z}\right)$ by the vanishing $i$-th cohomology of $\mathcal{O}_{Y}, i>0$. Since $Z$ is affine, there is a regular function $r$ on $Z$ such that $\left.r\right|_{F}$ is not a constant. Lift this function to $Y$, we are done. 
Step 4. The algebraic Stein variety $Y$ with $\kappa(D, X)=d=\operatorname{dim} Y$ is quasi-affine, that is, $Y$ is a Zariski open subset of an affine variety.

Proof. This part of proof is due to Goodman and Hartshorne [GH].

By a result of Goodman and Hartshorne [GH], since the claim of Step 3 is true, there is a proper morphism $\xi: Y \rightarrow U$ to a quasi-affine variety $U$. Since $Y$ is Stein, $Y$ contains no complete curves. So the fiber of the map $\xi$ is of 0 dimensional and finite. Therefore $\xi$ is a quasi-finite morphism. By Zariski's Main Theorem ([Mu1], Chapter III, Section 9), $\xi$ factors through an open immersion $\alpha: Y \rightarrow V$ followed by a finite morphism $\beta: V \rightarrow U$. So $Y$ is a quasi-affine variety.

Since $Y$ is quasi-affine and $H^{i}\left(Y, \mathcal{O}_{Y}\right)=0$ for all $i>0$, by Neeman's Theorem ([N], Theorem 4.1), $Y$ is affine.

Now we have completed the proof of Theorem 3.1.

Q.E.D.

Theorem 3.3. If $Y$ is an irreducible quasi-projective Stein variety with dimension $d$, then $\kappa(D, X) \neq d-1$.

Proof. If $Y$ is a curve, then $Y$ is affine since it is not complete ([H2], Chapter II, Proposition 4.1; [N]). Choose sufficiently large $n$ such that $n D$ is ample. The ampleness implies $\kappa(D, X)=\kappa(n D, X)=1$. So the theorem holds for curves.

Suppose that for all $(d-1)$-dimensional varieties the theorem is true. Let $\operatorname{dim} Y=$ $d>1$

If $h^{0}\left(X, \mathcal{O}_{X}(m D)\right)=1$ for all $m \geq 0$, then $\kappa(D, X)=0$. Since $D$ is effective, we may assume that $h^{0}\left(X, \mathcal{O}_{X}(m D)\right)>1$ for all $m \gg 0$. Then we have the fiber space defined by a nonconstant regular function on $Y$ as in the proof of Theorem 3.1. As before we have the following commutative diagram

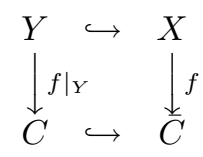

where $C$ is a smooth affine curve embedded in a smooth projective curve $\bar{C}, f$ is proper and surjective and every fiber of $f$ over $\bar{C}$ is connected. Let $\pi: X^{\prime} \rightarrow X$ be a proper and surjective birational morphism such that $X^{\prime}$ is smooth. Then we have ([Uen], Chapter II, Theorem 5.13)

$$
\kappa(D, X)=\kappa\left(\pi^{*} D, X^{\prime}\right) .
$$

To compute $\kappa(D, X)$, we may assume that $X$ is smooth in the above commutative diagram as in the proof of Theorem 3.1, Step 1.

Let $t_{1}$ be a point in $\bar{C}-C$, then $\kappa\left(t_{1}, \bar{C}\right)=1$. For a general point $t \in C$, we have $\kappa(t, \bar{C})=1$. By the Riemann-Roch formula, there is a positive integer $m$, such that

$$
h^{0}\left(\bar{C}, \mathcal{O}\left(m t_{1}-t\right)\right)>1 .
$$

Let $s$ be a nonconstant section of $H^{0}\left(\bar{C}, \mathcal{O}\left(m t_{1}-t\right)\right)$, then

$$
\operatorname{div} s+m t_{1}-t \geq 0 \text {. }
$$

Pull it back to $X$, we have

$$
f^{*}\left(\operatorname{div} s+m t_{1}-t\right)=\operatorname{div} f^{*}(s)+m f^{*}\left(t_{1}\right)-f^{*}(t) \geq 0 .
$$


Let $D_{1}=f^{*}\left(t_{1}\right)$ and $F=f^{*}(t)$, then

$$
h^{0}\left(X, \mathcal{O}_{X}\left(m D_{1}-F\right)\right)>1 .
$$

Choose an effective divisor $D$ with support $X-Y$ such that $D_{1} \leq D$, then we have

$$
h^{0}\left(X, \mathcal{O}_{X}(m D-F)\right) \geq h^{0}\left(X, \mathcal{O}_{X}\left(m D_{1}-F\right)\right)>1 .
$$

We know that the fibre $F$ is a prime vertical divisor and $f(D)=\bar{C}$. This implies that $\left.D\right|_{F}$ is an effective divisor on $F$. Since $\left.F\right|_{Y}$ is a Stein subvariety of dimension $d-1$ ([Gu], Page 143), by the inductive assumption, $\kappa\left(\left.D\right|_{F}, F\right) \neq d-2$. By Fujita's equation,

$$
\kappa(D, X)=\kappa(m D, X)=\kappa\left(\left.m D\right|_{F}, F\right)+\kappa(t, \bar{C}) \neq(d-2)+1=d-1 .
$$

Q.E.D.

Example 3.4. Notation is the same as in Theorem 3.3.

If $d=2 k$, then $\kappa(D, X)$ can be any even number $i, i=0,2,4, \ldots, 2 k$.

$D$ is effective, so $\kappa(D, X) \geq 0$. If $d=2$, then $k=1$ and $\kappa(D, X)$ can be 0 or 2 but not 1 by Corollary 2.8. Suppose that the equation holds for $(2 k-2)$-dimensional varieties $V, k>1$. We may assume that $V$ is smooth. We will construct an $(2 k)$ dimensional variety $Y$ with the prescribed $D$-dimension $i$, an even number.

Let $C$ be an elliptic curve (projective) and $E$ the unique nonsplit extension of $\mathcal{O}_{C}$ by itself. Let $Z=\mathbb{P}_{C}(E)$ and $F$ be the canonical section, then $S=Z-F$ is a Stein surface $([\mathrm{H} 2]$, Chapter VI, Example 3.2) and $\kappa(F, Z)=0[\mathrm{Ku}]$. Let $A$ be a smooth affine curve. Let $Y=V \times S, X=\bar{Y}$ be a smooth completion of $Y$ and $D$ be an effective divisor with support $X-Y$. The transcendental degree of $H^{0}\left(X, \mathcal{O}_{X}(m D)\right)$ does not change since $S$ has no nonconstant regular functions. In fact, by Künneth formula,

$$
\begin{gathered}
\kappa(D, X)=\operatorname{tr} \operatorname{deg}_{\mathbb{C}} \oplus_{m \geq 0} H^{0}\left(X, \mathcal{O}_{X}(m D)\right)-1 \\
=\operatorname{tr} . \operatorname{deg}_{\mathbb{C}} \oplus_{m \geq 0} H^{0}\left(\bar{V}, \mathcal{O}_{\bar{V}}\left(\left.m D\right|_{\bar{V}}\right)\right)-1=\kappa\left(\left.D\right|_{\bar{V}}, \bar{V}\right),
\end{gathered}
$$

where $\bar{V}$ is the projective variety containing $V$. If $Y=V \times A \times A$, then

$$
\begin{gathered}
\kappa(D, X)=\text { tr.deg } \operatorname{de}_{\mathbb{C}} \oplus_{m \geq 0} H^{0}\left(X, \mathcal{O}_{X}(m D)\right)-1 \\
=\text { tr.deg }_{\mathbb{C}} \oplus_{m \geq 0} H^{0}\left(X, \mathcal{O}_{\bar{V}}\left(\left.m D\right|_{\bar{V}}\right)\right)-1+2=\kappa\left(\left.D\right|_{\bar{V}}, \bar{V}\right)+2 .
\end{gathered}
$$

By the inductive assumption, the claim holds for $Y$.

Example 3.5. If $d=2 k+1$, then $\kappa(D, X)$ can be any odd number $j, j=1,3,5, \ldots$, $2 k-1,2 k+1$.

The calculation is the same as above. We start with a Stein curve $C$. Let $X=\bar{C}$ be the complete curve containing $C$, let $D$ be the boundary divisor with support $\bar{C}-C$, then $\kappa(D, X)=1$. Thus the claim holds for curves. Suppose that the equation holds for $(2 k-1)$-dimensional Stein variety $V, k \geq 1$. Let $Y$ be a Stein variety with dimension $2 k+1$. If we define $Y$ to be the product space by adding two affine curves, i.e., if $Y=V \times C \times C$, then the dimension of the variety increases by 2 and the $D$-dimension also increases by 2 . If $Y=V \times S$, then the dimension of the variety increases by 2 but the $D$-dimension does not change. 
Remark 3.6. 1. I do not know whether there exists an algebraic Stein threefold $Y$ such that $Y$ has no nonconstant regular functions, i.e., $H^{0}\left(Y, \mathcal{O}_{Y}\right)=\mathbb{C}$.

2. If $Y$ is a quasi-projective Stein variety with dimension $2 k$, then $\kappa(D, X) \neq 2 k-1$. I do not know whether $\kappa(D, X)=i, i=2 k-3,2 k-5, \ldots, 3,1$ is possible or not.

3. Similarly, if $Y$ is a quasi-projective Stein variety with dimension $2 k+1$, then $\kappa(D, X) \neq 2 k$. I do not know whether $\kappa(D, X)=i, i=2 k-2,2 k-4, \ldots, 2,0$ is possible or not.

Theorem 1.3 has been proved.

Corollary 3.7. If $Y$ is an irreducible algebraic Stein variety with dimension $d \geq 1$, then the following conditions are equivalent

(1) $Y$ is affine;

(2) $Y$ is regularly separable and $H^{i}\left(Y, \mathcal{O}_{Y}\right)=0$ for all $i>0$;

(3) $Y$ is regularly separable and $\Gamma\left(Y, \mathcal{O}_{Y}\right)$ is a finitely generated $\mathbb{C}$-algebra;

(4) $\kappa(D, X)>\max (1, d-2)$ and $H^{i}\left(Y, \mathcal{O}_{Y}\right)=0$ for all $i>0$;

(5) $\kappa(D, X)=d$, and $H^{i}\left(Y, \mathcal{O}_{Y}\right)=0$ for all $i>0$.

Proof. First, by Theorem 3.3, claim (4) and (5) are equivalent.

$(1) \Rightarrow(2) \Rightarrow(5) \Rightarrow(1)$ : We only need to prove $(2) \Rightarrow(5)$, that is, if $Y$ is regularly separable, then $\kappa(D, X)=d$.

If $Y$ is a curve, then $Y$ is affine so $\kappa(D, X)=1$. Suppose the claim holds for any $(d-1)$-dimensional variety. Since $Y$ is regularly separable, there is a nonconstant regular function $f$ on $Y$. As in the proof of Theorem 3.1, given by this function, there is a commutative diagram

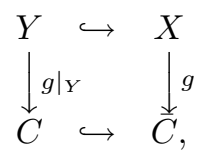

such that every fiber $X_{t}=g^{-1}(t)$ is connected and general fiber is irreducible. As in Step 1, proof of Theorem 3.1, we may assume that $X$ is smooth. Let $D_{t}=\left.D\right|_{X_{t}}$. By Theorem 2.1, we have

$$
\kappa(D, X)=\kappa\left(D_{t}, X_{t}\right)+1=(d-1)+1=d .
$$

$(3) \Rightarrow(1)$ : If $Y$ is Stein and regularly separable, then $\kappa(D, X)=d$ so $Y$ is quasiaffine by the proof of Step 4, Theorem 3.1. Since $\Gamma\left(Y, \mathcal{O}_{Y}\right)$ is a finitely generated $\mathbb{C}$-algebra, $Y$ is affine ([N], Proposition 5.5).

$(1) \Rightarrow(3)$ : Trivial.

The proof is completed.

Q.E.D.

\section{References}

[Art] M. Artin, On isolated rational singularities of surfaces, Amer. J. Math. 88 (1966), 129-136.

[Ba] L. Bădescu, Algebraic Surfaces, Universitext, Springer-Verlag, 2001.

[Fuj1] T. Fujita, On Zariski problem, Proc. Japan Acad. Ser. A Math. Sci. 55 (1979), no. 3, 106-110

[Fuj2] _ Some remarks on Kodaira dimension of fiber spaces, Proc. Japan Acad. 53, Ser. A, No.1 (1977).

[GH] J. Goodman, and R. Hartshorne, Schemes with finite-dimensional cohomology groups, American Journal of Mathematics, 91 (1969), no. 1, 258-266. 
[Gu] R. C. Gunning, Introduction to holomorphic functions of several variables, Vol. III. Homological theory, 1990

[H1] R. Hartshorne, Algebraic Geometry, Springer-Verlag, 1997.

[H2] - Ample Subvarieties of Algebraic Varieties, Lecture Notes in Mathematics, 156, Springer-Verlag, 1970.

[I1] S. Iitaka, On D-dimensions of algebraic varieties J. Math. Soc. Japan 23 (1971) 356-373.

[I2] , Birational Geometry for Open Varieties, Les Presses de l'Universite de Montreal, 1981.

[I3] B Birational geometry and logarithmic forms, Recent Progress of Algebraic Geometry in Japan, North-Holland Mathematics Studies 73, 1-27.

[Kl] S. L. Kleiman, Toward a numerical theory of ampleness, Ann. of Math. 84 (1966), no. 3, 293-344.

[KM] J. Kollár and S. Mori, Birational Geometry of Algebraic Varieties, Cambridge University Press, 1998.

[Ku] N. Mohan Kumar, Affine-Like Surfaces, Journal of Algebraic Geometry, 2 (1993), no. 4, 689-703.

[Mo] S. Mori, Classification of higher-dimensional varieties, Algebraic geometry, Bowdoin, 1985 (Brunswick, Maine, 1985), 269-331, Proc. Sympos. Pure Math., 46, Part 1, Amer. Math. Soc., Providence, RI, 1987.

[Mu1] D. Mumford, The Red Book of Varieties and Schemes, Lecture Notes in Mathematics 1358, Springer-Verlag, 1988.

[Mu2] _ The topology of normal singularities of an algebraic surface and a criterion for simplicity, Inst. Hautes tudes Sci. Publ. Math. No. 9, 1961, 5-22.

[N] A. Neeman, Steins, affines and Hilbert's fourteenth problem, Ann. of Math. (2) 127 (1988), no. 2, 229-244.

[O1] T. Ohsawa, A remark on pseudoconvex domains with analytic complements in compact Kälher manifolds, J. Math. Kyoto Univ. 47 (2007), no. 1, 115-119.

[O2] $-\bar{\partial}$-cohomology and geometry of the boundary of pseudoconvex domains, Ann. Polon. Math. 91 (2007), no. 2-3, 249-262.

[Sa1] F. Sakai, D-dimensions of algebraic surfaces and numerically effective divisors, Compositio Math. 48 (1983), no. 1, 101-118.

[Sa2] _ Weil divisors on normal surfaces, Duke Math. J. 51 (1984), no. 4, 877-887.

[SaW] J. H. Sampson and G. Washnitzer, A Künneth formula for coherent algebraic sheaves, Illinois J. Math. 3 (1959), 389-402

[Sei] A. Seidenberg, The hyperplane sections of normal varieties, Trans. Amer. Math. Soc. 69 (1950), 357-386.

[Sh] I. R. Shafarevich, Basic algebraic geometry I, Springer-Verlag, Berlin, 1994.

[Uen] K. Ueno, Classification Theory of Algebraic Varieties and Compact Complex Spaces, Lecture Notes in Mathematics, 439, 1975, Springer-Verlag.

[Za] O. Zariski, The theorem of Riemann-Roch for high multiples of an effective divisor on an algebraic surface, Ann. of Math. (2), 76 (1962), 560-616.

[Zh1] J. Zhang, Threefolds with vanishing Hodge cohomology, Trans. Amer. Math. Soc. 357 (2005), no. 5, 1977-1994.

[Zh2] _ There exist nontrivial threefolds with vanishing Hodge cohomology, Michigan Math. J. 54 (2006), 447-467.

[Zh3] _ Affine Algebraic Varieties, preprint.

Department of Mathematics and Statistics, University at Albany, SUny, Albany, Ny 12222, USA

E-mail address: jzhang@albany.edu 